\title{
January 2016 Critical Care Case of the Month
}

\author{
Sandra L. Till, DO \\ Banner University Medical Center Phoenix \\ Phoenix, AZ USA
}

\section{History of Present IIIness}

The patient is an 18-year-old woman who was driving to high school on a frontage road when she fell asleep at the wheel and her car rolled over. She was wearing her seatbelt but there was no airbag deployment. She did not lose consciousness and she was responsive and answering questions at the scene. She self-extricated from the vehicle. She had left arm pain with a boney deformity and she walked to the ambulance that transferred her to the hospital emergency department (ED).

Upon arrival in the ED she appeared pale and had difficulty breathing. In addition to her arm pain with an obvious left humeral fracture she also complained of upper abdominal and anterior chest pain. O2 saturation was initially $90 \%$ but declined to $70 \%$.

Which of the following should be ordered immediately?

1. Begin intravenous lines with large bore needles

2. X-ray of humerus

3. Hemoglobin and hematocrit

4. 1 and 3

5. All of the above 


\section{Correct!}

\section{1 and 3}

Initial assessment of a trauma victim is directed by the acronym ABCDE (1) where:

- A stands for Airway

- B stands for Breathing

- C stands for $\underline{\text { Circulation }}$

- D stands for Disability and neurological Damage (Make a rapid neurological assessment -is the patient awake, vocally responsive to pain or unconscious?)

- E stands for Exposure (Undress the patient and look for injury. If you suspect a neck or spinal injury, in-line immobilization is important)

The patient is pale suggesting potential blood loss. The declining oxygen saturation suggests possible trauma to the chest. Although the humeral fracture will need to be dealt with at some point, potential blood loss and chest trauma can be life-threatening and their evaluation should receive priority. A chest x-ray was taken but the patient arrested just after completion of the x-ray. She was intubated, but unfortunately vomited and aspirated during intubation. After several minutes of cardiopulmonary resuscitation spontaneous circulation returned. The chest x-ray became available (Figure 1).

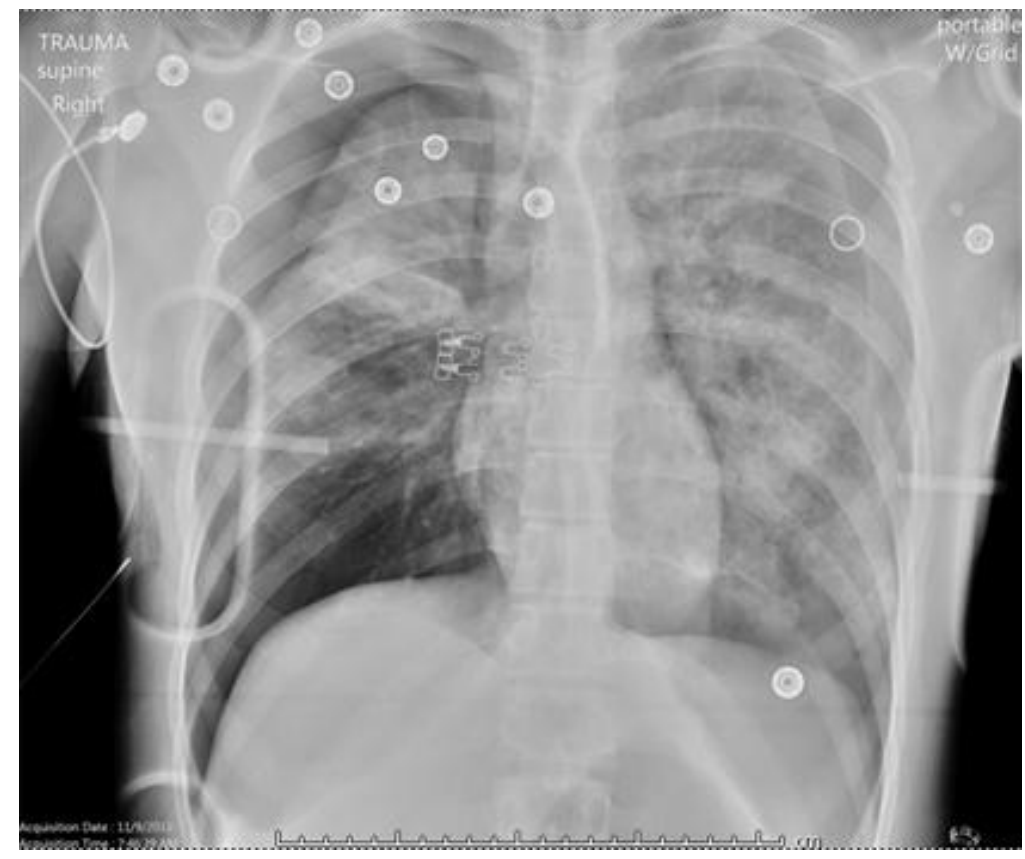

Figure 1. Initial portable AP of chest taken immediately before cardiac arrest.

Which of the following should be done next?

1. Administer high dose corticosteroids for aspiration pneumonia

2. Bronchoscopy to remove vomitus from the airways

3. Insert bilateral thoracostomy tubes

4. 1 and 3

5. All of the above 


\section{Correct! \\ 3. Insert bilateral chest tubes}

The chest $\mathrm{x}$-ray shows bilateral pneumothoracies right greater than left. After insertion of bilateral thoracostomy tubes her SpO2 improved to over $95 \%$ on a $\mathrm{FiO} 2$ of $100 \%$. However, also note that the lungs do not appear normal. There appears to be diffuse consolidation. Corticosteroids do not prevent development of aspiration pneumonia. Bronchoscopy with bronchoalveolar lavage can be done to remove any large airway food particles, but unless clinically suspected to result in tracheal or major lobar obstruction, would have little role in this acute situation.

The patient's initial laboratory returned and is below:

- White Blood Cell Count: $9.3 \times 10^{3}$ cells $/ \mathrm{mcL}$

- Hemoglobin: $9.3 \mathrm{~g} / \mathrm{dL}$

- Hematocrit: $26.3 \%$

- $\mathrm{Na}^{+}: 151 \mathrm{mEq} / \mathrm{L}$

- $\mathrm{K}^{+}: 3.5 \mathrm{mEq} / \mathrm{L}$

- $\mathrm{Cl}^{-}: 120 \mathrm{mEq} / \mathrm{L}$

- $\mathrm{HCO}^{-}: 8 \mathrm{mEq} / \mathrm{L}$

- Arterial blood gases (ABGs): pO2 75, pCO2 41, pH 7.36 on an FiO2 of 100\%, positive end expiratory pressure (PEEP) $20 \mathrm{~cm} \mathrm{H} 2 \mathrm{O}$

Which of the following should be done next?

1. Begin proning

2. CT scan of the chest, abdomen, and pelvis with contrast

3. Extracorporeal membrane oxygenation (ECMO)

4. 1 and 3

5. All of the above 


\section{Correct!}

\section{CT scan of the chest, abdomen, and pelvis with contrast}

Although the high $\mathrm{FiO} 2$ with relatively low pO2 is concerning, the major life-threatening threat is hemorrhage. In the setting of a motor vehicle accident with major blunt trauma this might suggest intra-abdominal hemorrhage such as a lacerated spleen or intrathoracic trauma such as aortic rupture. Although there is no widening of the mediastinum on the chest $\mathrm{x}$-ray ( $>6 \mathrm{~cm}$ upright, $>8 \mathrm{~cm}$ supine), an aortic rupture is still possible. The quickest diagnostic procedure is chest and abdominal CT scans in hemodynamically stable patients (2). She was judged sufficiently stable to undergo CT scanning. The chest CT revealed no aortic injury but the abdominal CT scan revealed a Grade V splenic injury (the worst injury). A CT scan of the head and spine performed at the same time showed no abnormalities. She was taken to the operating room and a splenectomy was performed along with her humeral fracture being reduced and set.

She returns to the intensive care unit post-operatively still on an FiO2 of $100 \%$ with 20 $\mathrm{cm} \mathrm{H} 2 \mathrm{O}$ PEEP with the following ABGs:

- pO2: 55

- pCO2: 41

- $\mathrm{pH}: 7.36$

Which of the following should be done next?

1. Corticosteroids for probably fat embolism

2. Extracorporeal membrane oxygenation (ECMO)

3. Recruitment maneuvers to lower the FiO2

4. 1 and 3

5. All of the above 


\section{Correct! \\ 3. Recruitment maneuvers to lower FiO2}

Fat embolism is possible from a humeral fracture although more commonly seen from the long bones of the leg. However, The use of corticosteroids in fat embolism is controversial (3). She meets the criteria for the adult respiratory distress syndrome (ARDS). The probable cause is pulmonary contusion. Her FiO2 does eventually need to be lowered in order to prevent oxygen toxicity. Sometimes lowering the FiO2 results in minimal fall in the pO2, but unfortunately, that was unsuccessful in this case. Recruitment maneuvers to increase the $\mathrm{pO} 2$ should be initially tried because of the speed and ease of many of these maneuvers (4). These can include:

- Proning

- Nitric oxide

- Prostacyclin

- Oscillatory ventilation

Unfortunately, none of these were successful either. For this reason a trial of venousvenous ECMO was begun which raised the pO2 sufficiently to lower the FiO2 to $60 \%$ (4). After 9 days ECMO was discontinued.

What is the greatest risk to the patient's survival at this time?

1. Infection

2. Myocardial infarction

3. Pulmonary embolism

4. Pulmonary fibrosis secondary to oxygen toxicity

5. Respiratory failure 


\section{Correct! \\ 1. Infection}

A number of years ago the group in Seattle pointed out that late in ARDS the major cause of mortality was infection, often from a lung source (5). After about 2 weeks, she developed fevers and leukocytosis. Bronchoscopy revealed E. coli. Despite appropriate antibiotics she continued with worsening leukocytosis and fevers. A CT scan was performed revealing bilateral loculated pleural effusion (Figure 2) and she had minimal drainage from her chest tubes.

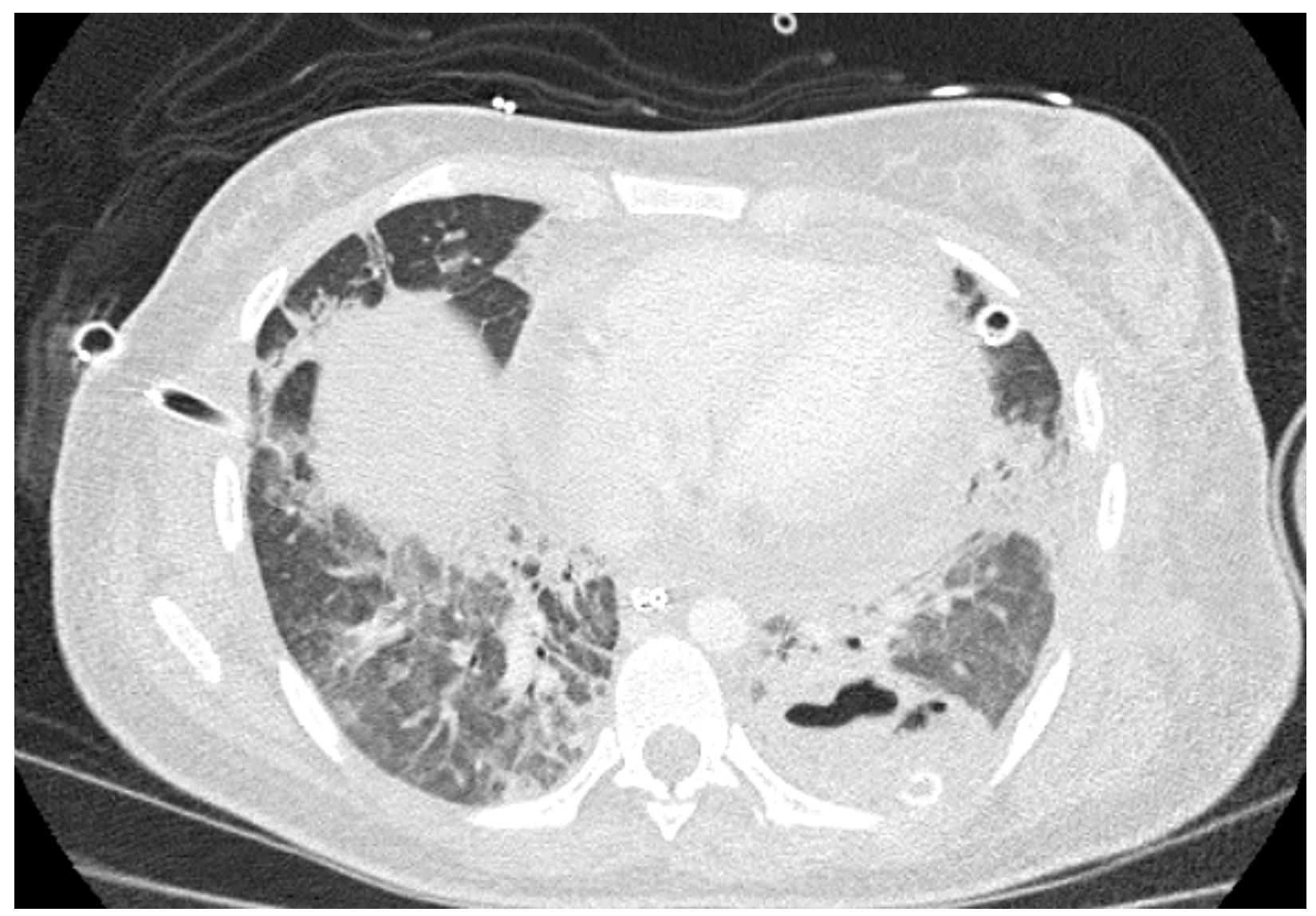

Figure 2. CT chest 2 for evaluation of fevers and leukocytosis two weeks after motor vehicle collision

Tissue plasminogen activator (TPA) was administered through the left chest tube drain into the area thought to be empyema without improvement in leukocytosis or fevers. A pigtailed catheter was inserted into the small right pleural effusion with instillation of TPA. Also a pericardial window and drainage of a pericardial effusion was performed. All cultures of fluid were negative. With drainage of the right loculated effusion and the pericardial effusion her leukocytosis and fevers improved. 
The pericardial effusion was thought to be secondary to inflammatory etiology due to open cardiac massage during Advanced Trauma Life Support in the initial cardiac arrest during presentation.

At this time she has had a tracheostomy and continues to receive mechanical ventilation in the ICU. However, it is anticipated that she will survive her ordeal, hopefully with little residual functional deficiency.

\section{References}

1. World Health Care Organization. Essential surgical care. Primary trauma care manual: ABCDE of trauma. Available on-line at: http://www.steinergraphics.com/surgical/manual01.html (accessed 12/4/15).

2. Mirvis SE, Shanmuganathan K. Trauma radiology: part I. Computerized tomographic imaging of abdominal trauma. J Intensive Care Med. 1994;9(3):151-63. [CrossRef] [PubMed]

3. Gupta A, Reilly CS. Fat embolism. BJA Education. 2007;7(5):148-151. [CrossRef]

4. Ried M, Bein T, Philipp A, Müller T, Graf B, Schmid C, Zonies D, Diez C, Hofmann HS. Extracorporeal lung support in trauma patients with severe chest injury and acute lung failure: a 10-year institutional experience. Crit Care. 2013;17(3):R110. [CrossRef] [PubMed]

5. Montgomery AB, Stager MA, Carrico CJ, Hudson LD. Causes of mortality in patients with the adult respiratory distress syndrome. Am Rev Respir Dis. 1985;132(3):485-9. [PubMed] 